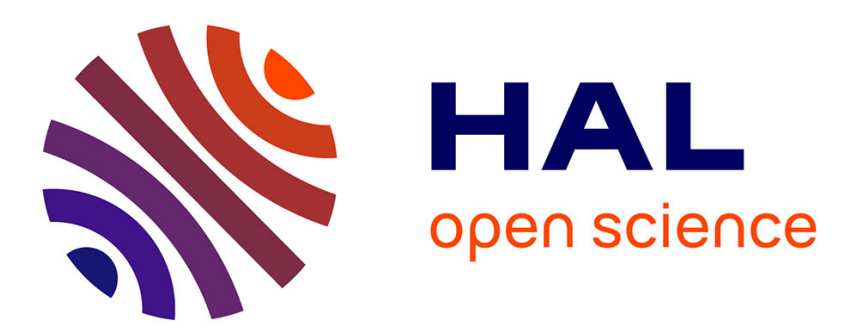

\title{
A Generalized Model for the Proton Expansion in Astrophysical Winds. I. The Velocity Distribution Function Representation
}

François Leblanc, Daniel Hubert

\section{- To cite this version:}

François Leblanc, Daniel Hubert. A Generalized Model for the Proton Expansion in Astrophysical Winds. I. The Velocity Distribution Function Representation. The Astrophysical Journal, 1997, 483 (1), pp.464-474. 10.1086/304232 . insu-02137410

\section{HAL Id: insu-02137410 https://hal-insu.archives-ouvertes.fr/insu-02137410}

Submitted on 23 May 2019

HAL is a multi-disciplinary open access archive for the deposit and dissemination of scientific research documents, whether they are published or not. The documents may come from teaching and research institutions in France or abroad, or from public or private research centers.
L'archive ouverte pluridisciplinaire HAL, est destinée au dépôt et à la diffusion de documents scientifiques de niveau recherche, publiés ou non, émanant des établissements d'enseignement et de recherche français ou étrangers, des laboratoires publics ou privés. 
The Astrophysical JouRnAL, 483:464-474, 1997 July 1

(C) 1997. The American Astronomical Society. All rights reserved. Printed in U.S.A.

\title{
A GENERALIZED MODEL FOR THE PROTON EXPANSION IN ASTROPHYSICAL WINDS. I. THE VELOCITY DISTRIBUTION FUNCTION REPRESENTATION
}

\author{
F. LEBLANC AND D. HUBERT \\ Département de Recherche Spatiale, CNRS URA 264, Observatoire de Paris \\ Received 1996 October 1; accepted 1997 January 28
}

\begin{abstract}
We construct a new approach to model the velocity distribution function (VDF) for the protons in stellar atmosphere expansions or planetary polar winds. The generalized Grad method of construction is used, and comparisons with the bi-Maxwellian polynomial expansion model are made in applications to the solar wind in the context of the measurements made by the Helios probes between 0.3 and $1 \mathrm{AU}$. A fitting procedure based on a sum of two Maxwellian functions is used to check the convergence property of both polynomial expansions and to calculate the predicted polynomial expansion profiles along the magnetic field orientation for typical proton VDFs in the solar wind. The generalized model is better adapted than the bi-Maxwellian polynomial expansion function to reproduce the long-tail features of a majority of the observed proton VDFs; moreover, our model does not display negative values of the VDF, contrary to the bi-Maxwellian expansion for normalized heat flux larger than unity. A 16 moment approximation, which corresponds to a third order of development, allows us to provide an associated set of generalized transport equations better closed than the equivalent system associated with a biMaxwellian polynomial expansion.
\end{abstract}

Subject headings: plasmas - solar system: general — solar wind - stars: mass loss

\section{INTRODUCTION}

The solar corona is the source region of the solar wind, a supersonic flow composed of electrons, protons, and heavy ions that carves the heliosphere. The polar wind is a plasma flow of electrons, protons, and $\mathrm{O}^{+}$ions, escaping from the polar terrestrial ionosphere along the magnetic field lines. Prototypes of stellar atmospheres expanding into the interstellar space, they expand from collision-dominated regions, through transition regions, to collisionless regions. To model such plasmas in their collision-dominated regions, an isotropic hydrodynamic approach (Chapman 1965; Parker 1958) is generally well adapted because the collisions between particles are sufficiently numerous to thermalize the flow. It consists of the resolution of a system of transport equations for the macroscopic parameters. In collisionless regions, kinetic models (Chamberlain 1960; Lemaire \& Scherer 1971) are much more adapted than fluid models. Indeed, particles in such a medium move nearly independently of the others. These methods generate exospheric models based on a microscopic description of the flow. But in transition regions, both models fail because, with a Knudsen number (which is defined as the ratio of the particle mean free path to the density scale height) roughly equal to one (Shizgal 1977), interactions between particles are sufficient to alter pure collisionless approaches and not enough to justify a pure collision-dominated state. For instance, the proton temperature anisotropy is equal to unity for all the hydrodynamic models, while the kinetic approaches provide proton temperature anisotropy $\left(T_{\|} / T_{\perp}\right)_{p}$ between 10 and 900 (Lemaire \& Scherer 1973), whereas observations in the solar wind reveal values of $2 \pm 1$.

Therefore, another approach is needed to model plasma expansions in these intermediate regions. Grad (1958) has proposed a solution of the Boltzmann equation, the socalled moment approach, in which the distribution function is a polynomial expansion with a weight function, which is the local equilibrium Maxwellian distribution function. Used in near-local-equilibrium states, it is based on the resolution of an associated system of transport equations for the velocity moments of the distribution function. Thus, Cuperman, Weiss, \& Dryer $(1980 ; 1981)$ have derived a set of transport equations up to the fourth order of the velocity moments for spatially inhomogeneous, anisotropic, multispecies, and spherically symmetric systems of particles obeying an inverse-square law of interactions. They have calculated the velocity collisional transfer by the FokkerPlanck operator based also on a local Maxwellian polynomial expansion. But for plasma flows in states far from the local equilibrium, such as, for instance, with large temperature anisotropies, these microscopic descriptions seem not to be available. Then, an improved solution is to take into account as first principles the properties of the nonequilibrium system. Chew, Goldgerger, \& Low (1956) were the first to derive transport equations based on a biMaxwellian species distribution function. Transport effects such as heat flux and viscosity for Coulomb collisions have been included by Chodura \& Pohl (1971) and Oraevskii, Chodura, \& Feneberg (1968). Finally, Demars \& Schunk (1979) extended this development to arbitrary degrees of ionization and built the 16 moment set of transport equations (using velocity moments until order 3 in gyrotropic microscopic states). They have shown in applications to the solar and polar winds (Demars \& Schunk 1989, 1990, 1991) the great interest of such models that provide a microscopic and a macroscopic description in relatively good agreement with observations.

Grad's solution cannot describe states with large temperature anisotropies because such a polynomial expansion based on an isotropic Maxwellian function is only allowed to generate small deviations from isotropic states. In the same way, a polynomial expansion with a bi-Maxwellian function as a weight factor cannot generate large deviations from the bi-Maxwellian distribution function. For instance, it cannot be used to describe plasma flows that are characterized by large, magnetic field-aligned heat flux because a bi-Maxwellian function is not well adapted to generate such 
heat flux. Several authors have tried to determine the fields of application of the Demars \& Schunk (1979) model. Palmadesso, Ganguli, \& Mitchell (1988) have found unstable waves modes when this model is used for largescale magnetospheric-ionospheric dynamics and have defined a threshold that depends on the magnetic fieldaligned heat flux $q_{\| p}$ normalized by the free-streaming heat flux (which is the thermal energy of ions $P_{\| p}$ times the magnetic field-aligned thermal speed $V_{\mathrm{th} \| p}$ ) and is $q_{\| p} \leq$ $0.44\left(P_{\|} V_{\mathrm{th} \|}\right)_{p}$. Above this limit the bi-Maxwellian expansion cannot correctly describe such plasmas. Cordier (1994a, 1994b) has studied the hyperbolic nature of the system of transport equations built from a 16 moment polynomial expansion for collisionless plasmas and has obtained very similar limits: $q_{\| p} \leq 0.46\left(P_{\|} V_{\text {th } \|}\right)_{p}$. More recently, a general analysis of the microscopic description was developed by Levermore, Morokoff, \& Nadiga (1995). It consists of studying the realizability and positivity of any model of velocity distribution functions (VDFs). They have built methods to make sure that the velocity moments and the VDF remain physically realistic. The best illustration of these rules is given by Gombosi \& Rasmussen (1991), who has shown that the 16 moment VDF has negative values for too high values of the magnetic field-aligned heat flux; Robineau, Blelly, \& Fontanari (1996) have stressed the hypothesis of fluid description and concluded that the transport of energy should contribute only to a small part of the local thermal energy creation. A different criterion has been defined by Mintzer (1965). He analyzed the nature of the convergence of any polynomial expansion generalizing the Grad approach and has obtained a necessary condition on the choice of the weight function in comparison with the exact solution. Then, Hubert (1985) has shown the nonconvergence of a bi-Maxwellian polynomial expansion describing a microscopic model characterized by a long tail, typical of the observed proton velocity distribution of the solar wind.

Considering all these analyses, the results of Demars \& Schunk (1990) are open to some criticisms because they have elaborated models for the solar wind proton VDF (Demars \& Schunk 1990) from Helios 2 solar probe data (Marsch et al. 1982). Indeed, this probe has revealed the existence of three main types of three-dimensional proton VDF contours: an isotropic state, an anisotropic state displaying a long tail in the magnetic field orientation, and a double-humped VDF oriented in the same way and associated with large values of temperature anisotropy and heat flux. Furthermore, the heat flux value is higher than the limit defined by previously cited authors [in $q_{\| p} \leq$ $\left.a\left(P_{\|} V_{\mathrm{th} \|}\right)_{p}\right]$ with the coefficient $a$ being larger than unity in most of these observed proton distributions, and the biMaxwellian polynomial expansion provides unphysical VDF profiles.

This paper is the first part of a work whose purpose is to establish a generalized microscopic solution as well as a generalized fluid theory for the description of timedependent, spatially nonhomogeneous, anisotropic, and multispecies space plasma winds. Our approach is directly inspired by the generalized polynomial solution of the Boltzmann equation established by Mintzer (1965), in which the most important point for application is the choice of the zeroth-order approximation. Indeed, it is as important that a good approximation can be obtained when the expansion is truncated after a small number of terms as it is for the expansion to converge when the number of terms approaches infinity; moreover, the zeroth-order approximations have to be sufficiently simple so as not to give a complex associated system of coefficients of the polynomial expansion (or, equivalently, a sufficiently simple associated system of the general transport equations). Thus, our approach will be to model the characteristics of the solar wind proton VDF in order to select a zeroth-order approximation from an exact solution of a similar but simplified problem. A similar step has been used by Hubert (1983) in the field of non-Maxwellian auroral phenomena, whose results provide good comparison with respect to Monte Carlo simulations (Hubert \& Barakat 1990; Barakat \& Hubert 1990).

Section 2 is devoted to the analysis of typical properties of the proton VDF observed in solar wind. Section 3 deals with the mathematical structures of the bi-Maxwellian polynomial expansion. In $\S 4$ we develop our new solution for the microscopic description of the proton in solar and polar winds. Section 5 is a brief discussion of the merits of this approach. A conclusion is presented in $\S 6$.

\section{CHARACTERISTICS OF THE PROTON VELOCITY DISTRIBUTION FUNCTION OF THE SOLAR WIND}

\subsection{Macroscopic Features}

Marsch et al. (1982) have provided observations of the radial evolution of the macroscopic parameters of the protons between 0.3 and $1 \mathrm{AU}$. Using the data of the Helios 2 probe, the evolution of the total temperature and the heat flux has been determined for different speeds of the protons. The total temperature $T_{p}$ evolves faster for low-speed winds than for high-speed winds. It follows a radial evolution as $R^{-\alpha}$, where $0.76 \leq \alpha \leq 1.33$, and $R$ is the distance to the Sun (Schwenn \& Marsch 1991). The proton temperature anisotropy $\left(T_{\|} / T_{\perp}\right)_{p}$ increases with heliocentric distance; it evolves between 1 and 2 and is always larger than unity at $1 \mathrm{AU}$. Furthermore, Marsch has shown that at $0.3 \mathrm{AU}$, the proton perpendicular temperature $T_{\perp p}$ could be twice as large as the proton parallel temperature $T_{\| p}$. Indeed, $T_{\perp p}$ decreases by 2 orders of magnitude from 0.3 to $1 \mathrm{AU}$. The evolution of the total heat flux $Q_{p}$ is also proportional to $R^{-\alpha}$, where $3.8 \leq \alpha \leq 4.7$. The heat flux is larger for slow winds than for fast ones. Finally, the perpendicular heat flux $q_{\perp p}$ is much smaller than the parallel heat flux $q_{\| p}$.

For heliocentric distances larger than $1 \mathrm{AU}$, data of several probes provide us macroscopic parameter radial variations also (Gazis 1984). The density, as expected, varies as $R^{-2}$, but the proton temperature evolves slower (following a $R^{-\alpha}$ law with $0.3 \leq \alpha \leq 0.7$; see Maksimovic 1995) than an adiabatic expansion, as it was the case between 0.3 and 1 AU. Gazis, from Voyager 1 data, has provided an estimation of the radial component of the heat flux. He has suggested that $Q_{p}$ follows a radial evolution as $R^{-2.44}$. The differences between observations made in the regions less distant than $1 \mathrm{AU}$ from the Sun and beyond are usually explained by a heating of the solar wind caused by shocks or interplanetary compression zones mainly observed beyond 1 AU. Moreover, Liu et al. (1995) have explained that somewhere near $10 \mathrm{AU}$, the observations of the macroscopic parameters are perturbed by the highspeed winds that mix with slow solar winds. Then, at large heliocentric distances, the analysis of the total proton temperature $T_{p}$ and of the total heat flux $Q_{p}$ have to take into 
account new cooling/heating mechanisms and interpenetrating processes.

To sum up, for all heliocentric distances, observations reveal a nonadiabatic expansion of an anisotropic plasma composed of particles that interact weakly. We will focus our attention on the evolution of the temperature anisotropy and the heat flux.

\subsection{Microscopic Features}

The study (Marsch et al. 1982; Marsch 1982) of the proton VDF has revealed some specific aspects of the solar wind microscopic state. It has shown a wide variety of nonthermal features: gyrotropic contours with respect to the magnetic field direction, a core and halo shape of the proton VDF along the magnetic field, a temperature anisotropy of the core always as $\left(T_{\|} / T_{\perp}\right)_{\text {core }} \leq 1$ while $\left(T_{\|} / T_{\perp}\right)_{\text {total }} \geq 1$ almost everywhere, large values of the magnetic fieldaligned heat flux, and even resolved double peaks. The observations have shown three main types of threedimensional VDFs. The first class is composed of isotropic VDFs and is mainly observed in interplanetary magnetic field sector boundaries. Most of the VDF profiles develop a long tail in the magnetic field orientation (see Fig. 2, below). The third class displays a surprising profile, which reveals a magnetic field-aligned second peak (see Fig. 1).

Observed at all heliocentric distances, this type of shape represents $20 \%$ of all the measurements made by the Helios 2 probe, according to Marsch et al. (1982). Furthermore, a correlation between the Alfvén speed and the relative speed between the two peaks persists during low and high streams, suggesting an Alfvén waves regulation phenomena (Montgomery et al. 1976). The double-humped proton distribution function is a stable feature of the solar wind proton VDF, related to large values of the magnetic fieldaligned heat flux.

The crosses in Figures 1 and 2 are a cut through the proton VDF cases I and $\mathrm{K}$ (obtained from Marsch data: Marsch et al. 1982) along the magnetic field direction. The case $\mathrm{K}$ was measured at an heliocentric distance of $0.39 \mathrm{AU}$ and a solar wind speed of $494 \mathrm{~km} \mathrm{~s}^{-1}$, and the case I at 0.54 AU and $618 \mathrm{~km} \mathrm{~s}^{-1}$.

In order to characterize the microscopic state, Feldman (1979) fits the observations with a sum of two Maxwellian functions. In Figures 1 and 2 we show results of such fitting. The dashed and dotted lines fit the core and the halo respec-

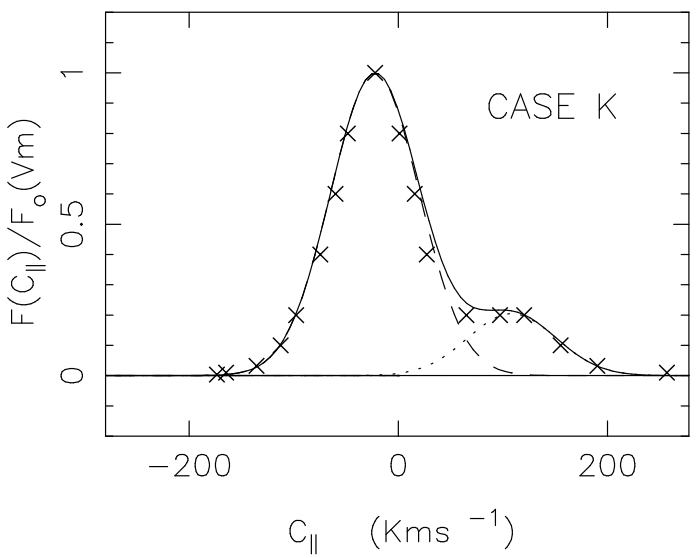

FIG. 1.-Magnetic field-aligned profile of case $\mathrm{K}$ issued from Marsch data and fitting by a sum of two Maxwellian functions plotted in the random velocity space. The crosses represent the Helios probe measurements, the solid line is the sum of two Maxwellian functions, and the dashed and dotted lines are the independent representation of the two Maxwellian functions composing the sum.

tively with a Maxwellian function, while the solid line is the sum of these two Maxwellian functions. Tables 1 and 2 give the value of the fitting variables obtained in the two cases I and $\mathrm{K}$ and the derived macroscopic parameters. It also provides the values determined by Marsch et al. (1982) for

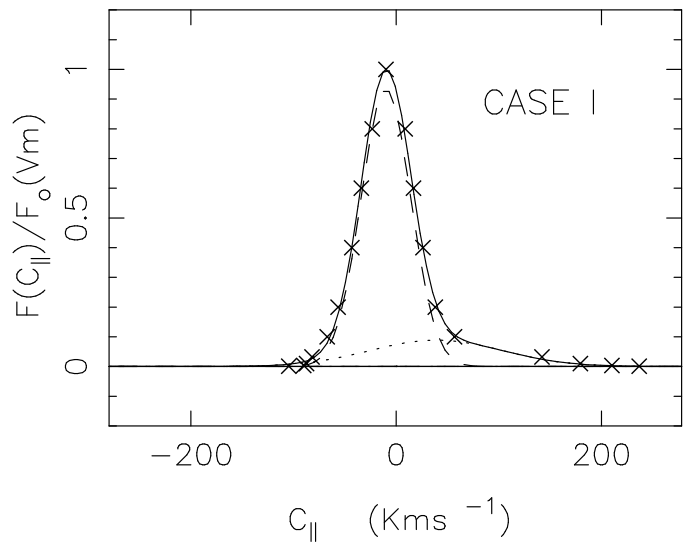

FIG. 2.-Magnetic field-aligned profile and fitting of case I: the plotting format is the same as that for Fig. 1 .

TABLE 1

FitTing of CASE I

\begin{tabular}{|c|c|c|c|c|}
\hline \multicolumn{2}{|c|}{ FITTING } & \multicolumn{3}{|c|}{ VeLOCITY MOMENTS } \\
\hline Parameter & Value & Parameter & Fitting Data & Marsch Data \\
\hline$n_{1} /\left(n_{1}+n_{2}\right)$ & 0.8 & $n_{p}\left(\mathrm{~cm}^{-3}\right)$ & 14 & 10.4 \\
\hline$T_{1}(\mathrm{~K})$ & 71,130 & $V_{m}\left(\mathrm{~km} \mathrm{~s}^{-1}\right)$ & 9.9 & $\ldots$ \\
\hline$T_{2}(\mathrm{~K})$ & 500,480 & $T_{\| p}$ & 204,420 & 267,000 \\
\hline \multirow{4}{*}{$U_{12}\left(\mathrm{~km} \mathrm{~s}^{-1}\right)$} & 49 & $q_{\| p} \times 10^{-13}\left(\mathrm{~kg} \mathrm{~m}^{3} \mathrm{~s}^{-3}\right)$ & 6.2 & 2.88 \\
\hline & & $n_{p} m_{p}\left\langle c_{\|}^{4}\right\rangle \times 10^{-8}\left(\mathrm{~kg} \mathrm{~m}^{4} \mathrm{~s}^{-4}\right)$ & 3.27 & $\ldots$ \\
\hline & & $n_{p} m_{p}\left\langle c_{\|}^{5}\right\rangle \times 10^{-3}\left(\mathrm{~kg} \mathrm{~m}^{5} \mathrm{~s}^{-5}\right)$ & 4.16 & $\ldots$ \\
\hline & & $n_{p} m_{p}\left\langle c_{\|}^{6}\right\rangle \times 10^{2}\left(\mathrm{~kg} \mathrm{~m}^{6} \mathrm{~s}^{-6}\right)$ & 8.08 & $\ldots$ \\
\hline
\end{tabular}

NoTE.-Results of a sum of two Maxwellian functions fitted to the profile along the magnetic field of case I. The terms $n_{1}, T_{1}$ defined the first Maxwellian function of the sum and $T_{2}, U_{12}$ the second; $U_{12}$ is the relative velocity between the two Maxwellian functions; $n_{2}=1-n_{1}, T_{1}$, and $T_{2}$ are temperatures; $V_{m}$ is the average velocity relative to the velocity of the maximum phase space density; $T_{\| p}$ is the parallel temperature; and $q_{\| p}$ is the parallel heat flux. 
TABLE 2

FitTing of CASE K

\begin{tabular}{|c|c|c|c|c|}
\hline \multicolumn{2}{|c|}{ FITTING } & \multicolumn{3}{|c|}{ VELOCITY MOMENTS } \\
\hline Parameter & Value & Parameter & Fitting Data & Marsch Data \\
\hline$n_{1} /\left(n_{1}+n_{2}\right)$ & 0.83 & $n_{p}\left(\mathrm{~cm}^{-3}\right)$ & 7.82 & 26.2 \\
\hline$T_{1}(\mathrm{~K})$ & 217,638 & $V_{m}\left(\mathrm{~km} \mathrm{~s}^{-1}\right)$ & 22.2 & $\ldots$ \\
\hline$T_{2}(\mathrm{~K})$ & 215,327 & $T_{\| p}$ & 509,706 & 566,000 \\
\hline \multirow[t]{4}{*}{$U_{12}\left(\mathrm{~km} \mathrm{~s}^{-1}\right)$} & 131 & $q_{\| p} \times 10^{-13}\left(\mathrm{~kg} \mathrm{~m}^{3} \mathrm{~s}^{-3}\right)$ & 3.47 & 6.19 \\
\hline & & $n_{p} m_{p}\left\langle c_{\|}^{4}\right\rangle \times 10^{-7}\left(\mathrm{~kg} \mathrm{~m}^{4} \mathrm{~s}^{-4}\right)$ & 9.92 & $\ldots$ \\
\hline & & $n_{p} m_{p}\left\langle c_{\|}^{5}\right\rangle \times 10^{-1}\left(\mathrm{~kg} \mathrm{~m}^{5} \mathrm{~s}^{-5}\right)$ & 1.05 & $\ldots$ \\
\hline & & $n_{p} m_{p}\left\langle c_{\|}^{6}\right\rangle \times 10^{+3}\left(\mathrm{~kg} \mathrm{~m}^{6} \mathrm{~s}^{-6}\right)$ & 2.25 & $\ldots$ \\
\hline
\end{tabular}

NotE.-The variables are defined as in Table 1.

comparison. It is important to underline that in our fitting we have only used a contour plot along one direction to calculate the macroscopic parameters, while Marsch has used three-dimensional contour plots. Furthermore, the value of $q_{\| p}$ that we give as the Marsch value is calculated as $q_{\| p}=q_{p} /\left(2 n_{p}\right)$, where $q_{p}$ is the heat flux density given in the Marsch data, and $n_{p}$ is the density.

As shown in Figures 1 and 2, a sum of two Maxwellians fits these profiles very well along the magnetic field direction. With this model, we are able to calculate the velocity moments at any order. We have established a great sensitivity of the profile in relation to the velocity moment values. Indeed, a variation of $10 \%$ of the heat flux value can generate a second peak in a profile modeled with a sum of two Maxwellian functions. The fitting has to be very accurate for regions of large velocity in order to obtain the best estimate of the velocity moments.

Figures $3 a$ and $3 b$ are obtained with the Marsch (1982) macroscopic parameters. They represent values of $q_{\| p}$ as a function of the parallel thermal velocity, $V_{\mathrm{th} \| p}=$ $\left(2 k_{B} T_{\| p} / m_{p}\right)^{1 / 2}$ times pressure term $P_{\| p}=k_{B} T_{\| p}\left(k_{B}\right.$ is the Boltzmann constant, and $m_{p}$ is the proton mass), which is the parallel free-streaming heat flux. The double-peaked VDF measurements (Fig. $3 b$ ) are separated into other cases. The solid line represents the relation $q_{\| p}=\left(P_{\|} V_{\mathrm{th} \|}\right)_{p}$. It corresponds to the upper limit defined in the paper of Palmadesso et al. (1988), above which unphysical behaviors

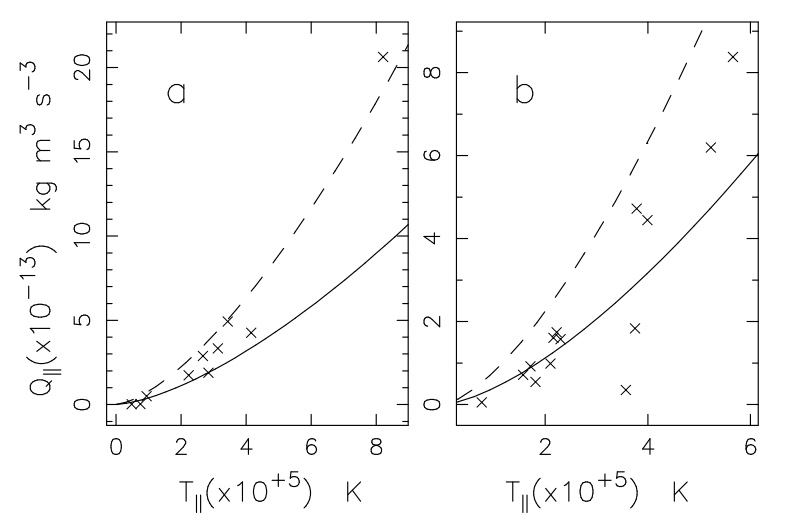

FIG. 3--Representation of $q_{\| p}$ as a function of $\left(P_{\|} V_{\mathrm{th} \|}\right)_{p}$ for Marsch values: $(a)$ the data without a resolved double peak; $(b)$ the double-humped cases. The crosses represent the Helios probe measurements, the solid line denotes the $q_{\| p}=\left(P_{\|} V_{\text {th } \|}\right)_{p}$ relation, and the dashed line denotes the constraints of construction of a generalized polynomial expansion. (for instance, negative values of the proton VDF) appear in the model of Demars \& Schunk (1979). Indeed, Palmadesso et al. (1988) have defined the domain of application of the solution of Demars \& Schunk (1979) by the condition $q_{\| p} \leq a\left(P_{\|} V_{\mathrm{th} \|}\right)_{p}$, where $a=0.44$ according to the study of the dispersion relation issued from a 16 moment model, and they have stressed that $a$ is equal to unity for dynamic fluid plasma simulations. We have retained this last value for $a$ as the less restrictive criterion. Although it is not a statistical analysis because of the small number of three-dimensional VDF profiles in Marsch's (1982) paper, it nevertheless gives an indication of the validity of the 16 moment model in solar wind. For instance, in cases I and $\mathrm{K}, q_{\| p} /\left(P_{\|} V_{\mathrm{th} \|}\right)_{p}$ is respectively equal to 1.7 and 1.3. Therefore, it seems that in most of these cases, the bi-Maxwellian polynomial expansion cannot be used to model solar wind proton VDF. Furthermore, we find no relation between the normalized parallel heat flux value and the existence of the double peak, if we compare Figure $3 a$ with Figure $3 b$.

The lack of measurements in transitional regions of the polar wind does not give us the possibility to provide a similar analysis. But a few Monte Carlo simulations (Barakat, Bargouthy, \& Schunk 1995; Wilson 1992) have supplied the evolution of the predicted proton VDF profiles. Thus, taking into account their results, we note that the typical proton VDF profiles in the polar wind are similar to the typical proton VDF shapes in the solar wind. Moreover, measurements in the solar wind are exclusively made in the transition region far from the Sun, while a number of measurements have been made in the collisiondominated region of the polar wind, but few are made in higher regions. Thus, the study of the polar wind has a complementary aspect to the study of the solar wind from the point of view of the measurements of the plasma parameters of astrophysical winds.

\section{THE BI-MAXWELLIAN POLYNOMIAL EXPANSION TO MODEL THE PROTON VELOCITY DISTRIBUTION FUNCTION}

\subsection{General Algebraic Rules of Construction}

First developed by Grad (1958), a polynomial expansion based on a weight function presents great interest for solving the Boltzmann equation. The weight function in the original Grad approach is the local Maxwellian equilibrium distribution function. His mathematical structure has been generalized for far-from-equilibrium states by Mintzer (1965). This latter has defined the general form of an expansion in velocity space around a zeroth-order distribution 
function, which has to be chosen as a good approximation in some limits of the exact solution of the Boltzmann equation (in order to ensure the rapid convergence of the expansion). The VDF is expressed as follows:

$$
f(\boldsymbol{r}, \boldsymbol{v}, t)=f^{0}(\boldsymbol{r}, \boldsymbol{v}, t) \sum_{q=0}^{+\infty} a_{q}(\boldsymbol{r}, t) M_{q}(\boldsymbol{v}),
$$

where $f^{0}$ is the zeroth-order function, the $M_{q}$ are a complete set of functions in velocity space (but may also depend on space and time), and the coefficients $a_{q}$ act as the generalized state variables.

In order to construct the simplest set of equations for the $a_{q}$, we define an orthonormalization rule,

$$
\int_{V} f^{0}(r, v, t) M_{p}(v) M_{q}(v) d v=\delta_{p q},
$$

which involves

$$
a_{q}(r, t)=\int_{V} f(r, v, t) M_{q}(v) d v
$$

where $V$ is the velocity space. The equations for $a_{q}$ are obtained by multiplying the Boltzmann equation by $M_{q}$ and integrating the result in velocity space. If only the first few velocity moments are useful, then a solution is to form the set $M_{q}$ from the complete set of monomials $1, v, v v, v v v$, ... (using a dyadic notation). Then, the moments of order $m$ will linearly derive from the coefficients $a_{q}$ with $q \leq m$. But the equation for $a_{q}$ involves coefficients of at least order $q+1$. Therefore, it is necessary to make some assumptions for the coefficients higher than those that we are solving. As for the Grad choice, Mintzer has suggested to truncate the expansion, that is, higher coefficients $a_{q}$ than a given order $m$ are set equal to zero. Finally, we obtain a closed set of equations for the coefficients $a_{q}$. In the same way, an equivalent system of equations for the velocity moments of lower order than $m$ (or for the equivalent macroscopic parameters) can be obtained by multiplying the Boltzmann equation with $v^{q}$ for $q$ varying from 0 to $m$ and by integrating in velocity space. The closure assumption is determined with the expression of $f$, which depends on $\left(a_{q}\right)_{q=0, m}$ and consequently on the velocity moments of lower order than $m$. Such a limiting condition, that is, to know a priori $f$, is essential in determining the expression of the collisional transfers for non-Maxwellian potential forces and in closing the set of transport equations in spatially nonhomogeneous systems.

Lowell (1967) has defined the convergence properties of the polynomial expansion constructed from equations (1), (2), and (3). We review them, as this point is seldom discussed, before embarking on long calculations that may not lead to a good approximation whatever the number of terms kept in the expansion. The coefficients $a_{q}$ of the expansion (eq. [3]) minimize the integral:

$$
I=\frac{1}{n_{p}} \int_{V} \frac{\left[f^{E}(\boldsymbol{r}, \boldsymbol{v}, t)-f^{A}(\boldsymbol{r}, \boldsymbol{v}, t)\right]^{2}}{f^{0}(\boldsymbol{r}, \boldsymbol{v}, t)} d \boldsymbol{v},
$$

where $f^{E}$ is the exact solution and $f^{A}$ the approximation. It is equivalent to minimizing the mean square error in an expansion of $F=f^{0-1 / 2} f^{E}$. Thus, the velocity space regions in which the $f^{0-1 / 2} f^{A}$ would be near $f^{0-1 / 2} f^{E}$, following this criterion, could not exactly correspond to the regions where the exact solution $f^{E}$ would be well approximated by $f^{A}$.
Therefore, particular care must be taken when we choose $f^{0}$. A natural criterion for the convergence of the polynomial expansion, which would have been that $\int_{V}\left[f^{E}(\boldsymbol{r}, \boldsymbol{v}, t)\right.$ $\left.-f^{A}(\boldsymbol{r}, \boldsymbol{v}, t)\right]^{2} d v$ must be minimized, is useless because it prevents an easy construction of the equations for the coefficients $a_{q}$ (Mintzer 1965). Then, a necessary condition derived from equation (4) is that the integral $\int_{V}\left[f^{E}(\boldsymbol{r}, \boldsymbol{v}, t)\right]^{2} /$ $\left[f^{0}(r, v, t)\right] d v$ exists. One condition for the definition of this integral is that

$$
\lim _{|\boldsymbol{v}| \rightarrow \infty} \frac{f^{E}(\boldsymbol{r}, \boldsymbol{v}, t)}{\left[f^{0}(\boldsymbol{r}, \boldsymbol{v}, t)\right]^{1 / 2}}=0 .
$$

In what follows we use the condition defined in equation (5) to know whether the polynomial expansion is divergent.

\subsection{Bi-Maxwellian Function as a Weight Factor}

Several authors have generalized Grad's method for farfrom-equilibrium states with a bi-Maxwellian function as a weight factor. First applied by Chew et al. (1956) for a collisionless anisotropic plasma, the approach was generalized by Demars \& Schunk (1979) to a large class of space plasmas. They have provided a system of transport equations for the first 16 velocity moments and have defined a microscopic description as in equation (1). The mathematical definition of the bi-Maxwellian weight function is

$$
\begin{aligned}
f_{b M}^{0}\left(r, c_{\|}, c_{\perp}, t\right)= & n_{p}\left(\frac{m_{p}}{2 k_{B} \pi T_{\| p}}\right)^{1 / 2} \frac{m_{p}}{2 k_{B} \pi T_{\perp p}} \\
& \times \exp \left\{-\frac{m_{p}}{2 k_{B} T_{\perp p}} c_{\perp}^{2}-\frac{m_{p}}{2 k_{B} T_{\| p}} c_{\|}^{2}\right\},
\end{aligned}
$$

where $n_{p}$ is the density, $c_{\|}$is the random thermal velocity parallel to the magnetic field $\left(c_{s}=v_{s}-u_{s}\right.$, where $\boldsymbol{u}_{\boldsymbol{s}}$ is the species average drift velocity), and the perpendicular velocity $c_{\perp}$ is a vector of two dimensions (we consider gyrationdominated plasmas), $T_{\| p}=m_{p}\left\langle c_{\| p}^{2}\right\rangle / k_{B}$ and $T_{\perp p}=$ $m_{p}\left\langle c_{\perp p}^{2}\right\rangle / 2 k_{B}$, where $\langle A\rangle=\int_{V} f(\boldsymbol{r}, \boldsymbol{v}, t) A d c_{p}$. The polynomials generated by the weight function defined in equation (6) and the orthonormalized rule (see eq. [2]) are the Hermite polynomials along the parallel velocity denoted $H_{i}$ and the associated Laguerre polynomials of order zero along the perpendicular velocity denoted $L_{j}$ (Jancel \& Kahan 1966). Therefore,

$$
\begin{aligned}
f_{b M}^{A}\left(r, c_{\|}, c_{\perp}, t\right)=f_{b M}^{0}\left(r, c_{\|}, c_{\perp}, t\right) \\
\times\left\{1+\sum_{i, j=1}^{m} a_{i j}(r, t) H_{i}\left(c_{\|}\right) L_{j}\left(c_{\perp}\right)\right\}
\end{aligned}
$$

is the approximation expansion of order $m$ with $i+j \leq m$. In the 16 moment development, $m$ is equal to 3 .

In the Demars \& Schunk (1990) investigations, the derived polynomial expansion should be able to reproduce all the profiles of the VDF observed in the solar wind and expected in the polar wind. Their model should also be able to provide the macroscopic parameter evolution in good agreement with observations. But some criticisms have been made on their conclusions. Palmadesso et al. (1988) have solved the dispersion relation issued from a 16 moment model and obtained unstable waves when $q_{\| p} /\left(P_{\|} V_{\text {th } \|}\right)_{p}$ is above about 0.44 . However, in their study they did not consider the collisional terms, and, consequently, they overestimated this criterion by neglecting dissipative terms. But they pointed out that in any case when running dynamic fluid plasma simulations, unstable behaviors appear when 

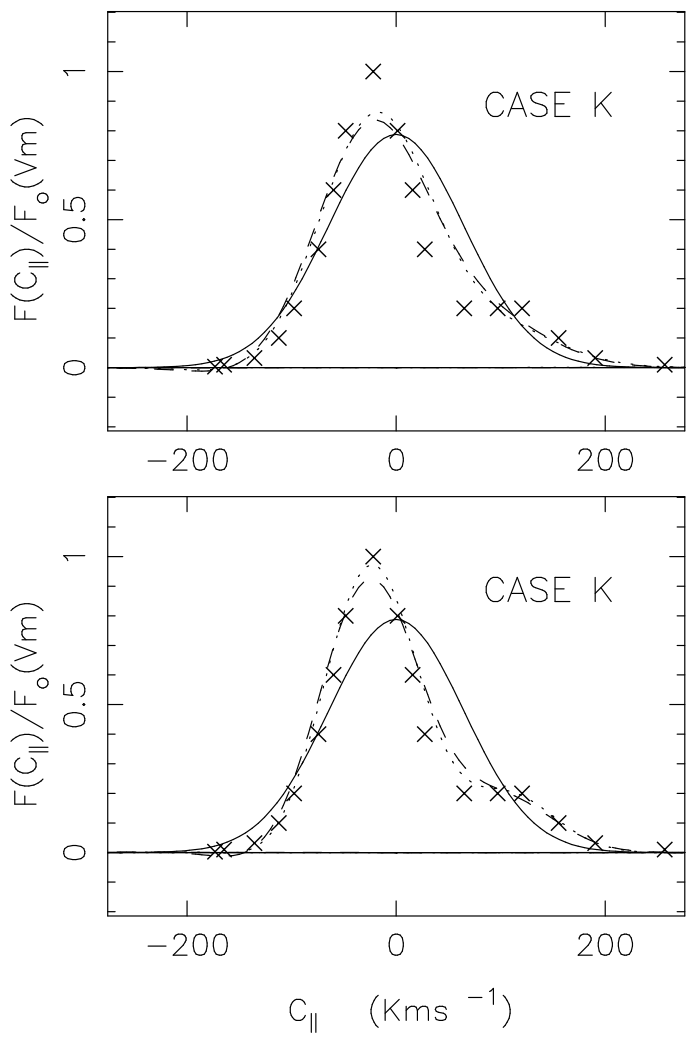

Fig. 4.-Polynomial expansion based on a bi-Maxwellian weight factor, centered on the average velocity for case $\mathrm{K}$ issued from Marsch data. The crosses represent the Helios probe measurements in the magnetic field direction. The solid line is the zeroth-order function defined in $\S 3.2$ (see eq. [6]). In the top panel, the dashed line corresponds to order 3 of development (see eq. [7]) and the dotted line to order 4, while the bottom panel shows orders 5 and 7 , respectively.

$q_{\| p} /\left(P_{\|} V_{\mathrm{th} \|}\right)_{p}$ is on the order of 1 . Indeed, in that case, the polynomial expansion $\Phi$ in $f_{b M}^{A}=f_{b M}^{0}(1+\Phi)$ reaches a value of the order of the unity, so that the VDF may be in the process of breaking down (Palmadesso et al. 1988). Thus, when Demars \& Schunk (1990) tried to reproduce contour plots of a typical proton VDF in the solar wind, in order to obtain a double-peaked shape, they increased the total proton heat flux value determined by Marsch within the important experimental uncertainty, and they arbitrarily fixed the anisotropy heat flux, which was not given in Marsch data. Therefore, their conclusions, to a certain extent, fail because they did not respect the limitation criterion on the parallel heat flux. Thus, the second peak appears simultaneously with negative values of the proton VDF. In conclusion, it seems that the contour plots with a double peak that they have obtained are due to a mathematical artifact of the polynomial expansion, as pointed out as early as 1985 by Hubert (Hubert 1985), stressing consequences on instabilities.

The convergence criterion of a polynomial expansion defined in equation (5) is not easy to apply because of the unknown $f^{E}$. But if we use the representation of $f^{E}$ defined in $\S 2.2$, then we are able to check such a criterion. We model the observed VDF by the sum of two Maxwellian distribution functions: $f(v)=n_{1} f_{\text {Max } 1}\left(T_{1}, v\right)+$ $n_{2} f_{\operatorname{Max} 2}\left(T_{2}, v\right)$. In regions of large velocity, one of the Maxwellian functions of the sum is much larger than the other,
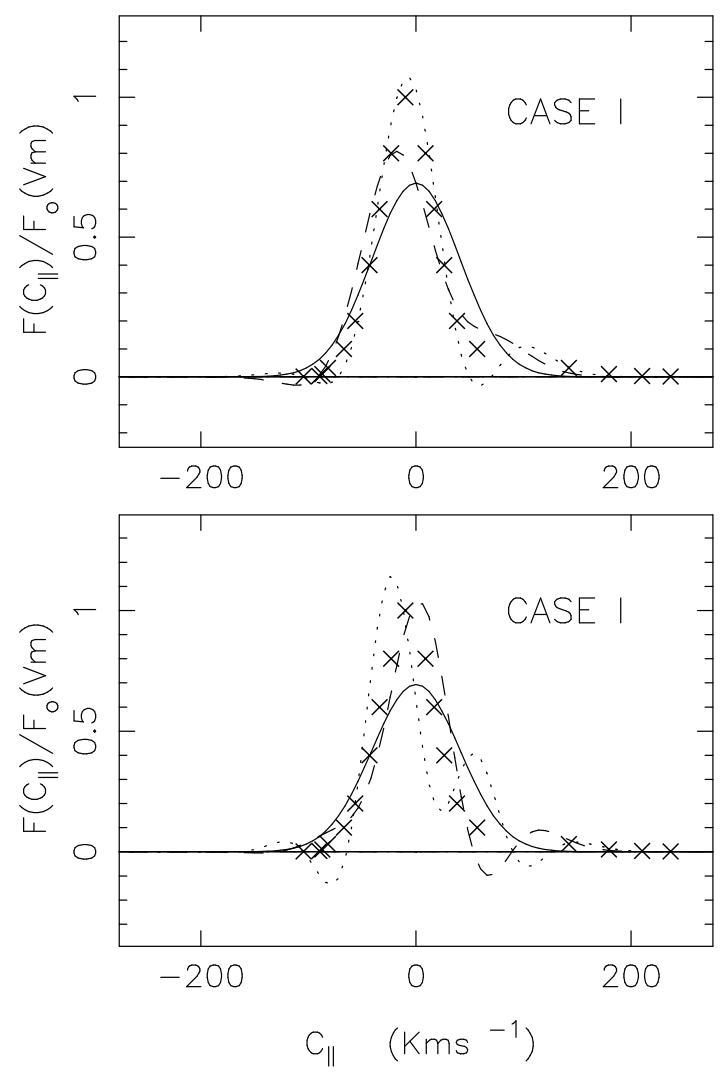

FIG. 5.-Polynomial expansion based on a bi-Maxwellian weight factor for case I: the plotting format is the same as that for Fig. 4.

which therefore can be neglected and is the function that fits the core. The convergence criterion (see eq. [5]) $f_{\text {Max }}\left(c_{\|}\right) /\left(f_{b M}^{0}\right)^{1 / 2}\left(c_{\|}\right) \rightarrow 0$ when $c_{\|} \rightarrow \pm \infty$ is then equivalent to the condition $T_{\mathrm{Max}}<2 T_{\|}$. Tables 1 and 2 give the fitting values (the Maxwellian function number 2 fits the halo, the number 1 the core); then, we can note that this required condition is not verified in case I for large positive velocity modulus, as $T_{\mathrm{Max}}=T_{2}=500,480 \mathrm{~K}$ and $2 T_{\|}=$ $408,840 \mathrm{~K}$.

In Figures 4 and 5 we have plotted Helios probe observations denoted as cases $\mathrm{K}$ and $\mathrm{I}$, respectively, in the parallel velocity plane (crosses) and the corresponding bi-Maxwellian polynomial expansion of order 0 (solid line), 3 (dashed line), and 4 (dotted line) in the top panel and 0 (solid line), 5 (dashed line), and 7 (dotted line) in the bottom panel. We note the evident nonconvergence of the model in case $\mathrm{I}$ and the slow convergence in case $\mathrm{K}$ (in that case we have established the convergence criterion and that the apparition of the oscillation is very sensitive to the values of the velocity moment). The convergence criterion defined in equation (5) seems to be realistic according to these two figures.

Furthermore, the negativity of the distribution function is in contradiction with the Demars \& Schunk (1979) assumption on the calculation of the collisional transfers, i.e., the polynomial expansion $\Phi$ must remain lower than unity.

\section{GENERALIZED POLYNOMIAL EXPANSION OF THE PROTON VELOCITY DISTRIBUTION FUNCTION IN POLAR AND SOLAR WINDS}

Mintzer (1965) has recommended the choice of a weight function (which is also the zeroth-order approximation) 
near the expected exact solution. Then, for applications to solar wind, an interesting idea for a microscopic model would be an expansion with a weight factor, such as an asymmetric function involving large parallel heat flux. Hubert (1985), being inspired by the Whealton \& Woo (1971) exact solution of the BGK equation for the moderate ionized plasma subjected to an electrostatic field, built a polynomial expansion with a weight function defined as

$$
\begin{aligned}
f_{G}^{0}\left(r, c_{\|}, c_{\perp}, t\right)= & n_{p} \frac{m_{p}}{4 k_{B} \pi T_{\perp p} D^{*}} \\
& \times \exp \left\{-\frac{m_{p}}{2 k_{B} T_{\perp p}} c_{\perp}^{2}-\frac{c_{\|}+D^{*}}{D^{*}}+\frac{1}{E^{*}}\right\} \\
& \times \operatorname{erfc}\left\{E^{* 1 / 2}\left(\frac{1}{E^{*}}-\frac{c_{\|}+D^{*}}{2 D^{*}}\right)\right\}
\end{aligned}
$$

where $c_{\|}, c_{\perp}$, and $T_{\perp p}$ were defined in $\S 3$, and erfc is the complementary error function (Abramovitz \& Stegun 1964). $D^{*}$ and $E^{*}$ are chosen such that the exact first-order velocity moments $\left\langle c_{\|}^{m}\right\rangle$ and $\left\langle c_{\perp}^{l}\right\rangle$ are equal to those provided by the zeroth-order approximation, that is,

$$
\begin{aligned}
& \int_{V} c_{\|}^{m} f_{G}^{0}(r, c, t) d c=\int_{V} c_{\|}^{m} f^{E}(r, c, t) d c \text { for } m \leq 3, \\
& \int_{V} c_{\perp}^{l} f_{G}^{0}(r, c, t) d c=\int_{V} c_{\perp}^{l} f^{E}(r, c, t) d c \text { for } l \leq 2 .
\end{aligned}
$$

Then, we obtain the following relations:

$$
\begin{aligned}
D^{*} & =\left(\frac{\left\langle c_{p \|}^{3}\right\rangle}{2}\right)^{1 / 3}, \\
E^{*} & =\frac{2 m_{p} D^{* 2}}{k_{B} T_{0}}, \\
T_{0} & =T_{\| p}-\frac{m_{p} D^{* 2}}{k_{B}},
\end{aligned}
$$

and the formal expression for $f_{G}^{A}$ in which $f_{G}^{0}$ is completely defined:

$$
\begin{aligned}
f_{G}^{A}\left(r, c_{\|}, c_{\perp}, t\right)= & f_{G}^{0}\left(r, c_{\|}, c_{\perp}, t\right) \\
& \times\left\{1+\sum_{i, j=1}^{n} a_{i j}(r, t) M_{i}\left(c_{\|}\right) L_{j}\left(c_{\perp}\right)\right\},
\end{aligned}
$$

where $\left(L_{j}\right)_{j=1, n}$ are Laguerre polynomials associated with the perpendicular part (dependent on $c_{\perp}$ ) of $f_{G}^{0} .\left(M_{i}\right)_{i=1, n}$ is an orthonormalized polynomial set defined in the $c_{\|}$velocity space and associated with the parallel part of $f_{G}^{0}$, and $n$ is the order of development with $i+j \leq n$. With this function we are able to calculate the velocity moments at any order.

Such a $f_{G}^{0}$ has a long suprathermal tail in the magnetic field direction and displays an anisotropic temperature. We follow the advice of Mintzer (1965) by choosing a zerothorder function as a known solution of equations that approximate the real physical phenomena. Indeed, in the polar wind Barakat et al. (1995) considered a flow of $\mathrm{H}^{+}$ ions through a background of $\mathrm{O}^{+}$ions (with constant average velocity and temperature) subjected to an electrostatic field and velocity-dependent Coulomb collisions. They obtained long-tailed VDF profiles that become double-humped VDF profiles when the number of collisions decrease in the transition region. In the solar wind a similar evolution of the VDF of the protons was obtained by Livi \& Marsch (1987) by studying the evolution of proton species subjected to self-Coulomb collisions described by the BGK operator. Therefore, the relaxation of protons in a background of neutrals subjected to an electrostatic field and a constant collision frequency (which is the problem solved by Whealton \& Woo 1971) can be considered as the first-order approximation of processes that provide typical observed VDFs.

Furthermore, the definition of $f_{G}^{0}$ proposed by Hubert imposes that $q_{\| p}$ be less than 2 times the parallel freestreaming flux (derived from the condition that the effective temperature $T_{0}$ has to be positive). This limitation is represented in Figure 3 by the dashed lines in the context of the observations made by the probe Helios in the solar wind. Most of the measurements are under the broken curve, which means that $f_{G}^{0}$ is almost everywhere defined. A similar approach for the study of non-Maxwellian ion states in the auroral latitudes has been developed by Hubert (1983). The polynomial expansion is based on the exact solution of the BGK equation for a moderate ionized plasma subjected to an electrostatic field perpendicular to the magnetic field. Hubert \& Barakat (1990) and Barakat \& Hubert (1990) have shown the good convergence properties of this polynomial expansion when compared to Monte Carlo simulations.

As the convergence property defined for a bi-Maxwellian function, an equivalent condition to equation (5) is obtained by substituting for large velocity modulus, $f^{E}$ with the sum of two Maxwellian distribution functions defined in $\S 2.2$. We have determined an equivalent condition as $T_{\text {Max }} \leq$ $2 T^{0}$, where $T^{0}$ is defined in equation (10), and $T_{\operatorname{Max}}$ is the larger temperature between the two temperatures $T_{1}$ and $T_{2}$ of the sum of the two Maxwellian functions. For case I, $2 T^{0}=91,105 \mathrm{~K}$ and $T_{\mathrm{Max}}=T_{2}=500,480 \mathrm{~K}$ for large negative values of the parallel velocity, and in case $K$, $2 T^{0}=484,182 \mathrm{~K}$ and $T_{\mathrm{Max}}=T_{1}=217,638 \mathrm{~K}$. However, for these regions we have observed that the function that fits the halo overestimates the profile of case I in large negative velocity regions; the other Maxwellian function is more adapted (in this case, the criterion of convergence is verified: $T_{\text {Max }}=T_{1}=71,133 \mathrm{~K}$ ). Nevertheless the long-tail part, in positive velocity regions, of the function is more interesting because it characterizes the non-Maxwellian behaviors of the VDF. Thus, for positive values of the parallel velocity, the convergence criterion is always verified. Indeed, the condition of convergence is equivalent to

$$
\lim _{c_{\| \rightarrow+\infty}} \frac{\exp \left[-\left(m_{p} / 2 k_{B} T_{\mathrm{Max}}\right) c_{\|}^{2}+\left(c_{\|} / D^{*}\right)\right]}{\operatorname{erfc}\left\{E^{* 1 / 2}\left[\left(1 / E^{*}\right)-\left(c_{\|}+D^{*}\right) / 2 D^{*}\right]\right\}^{1 / 2}}=0,
$$

which is always verified because

$$
\lim _{c_{\| \rightarrow+\infty}} \operatorname{erfc}\left[E^{* 1 / 2}\left(\frac{1}{E^{*}}-\frac{c_{\|}+D^{*}}{2 D^{*}}\right)\right]=2 .
$$

Therefore, the necessary convergence condition defined in $\S 3$ is verified in both cases.

We show in Figures 6 and 7 the corresponding polynomial expansion function $f_{G}^{A}$ obtained with velocity moments of Tables 1 and 2 in the same way as in Figures 4 and 5. We have used for the construction of $f_{G}^{A}$ the same order $m$ of development and the assumption of gyrationdominated plasmas as in $\S 3$. The crosses represent the Helios probe measurements. The top panel of Figures 6 and 

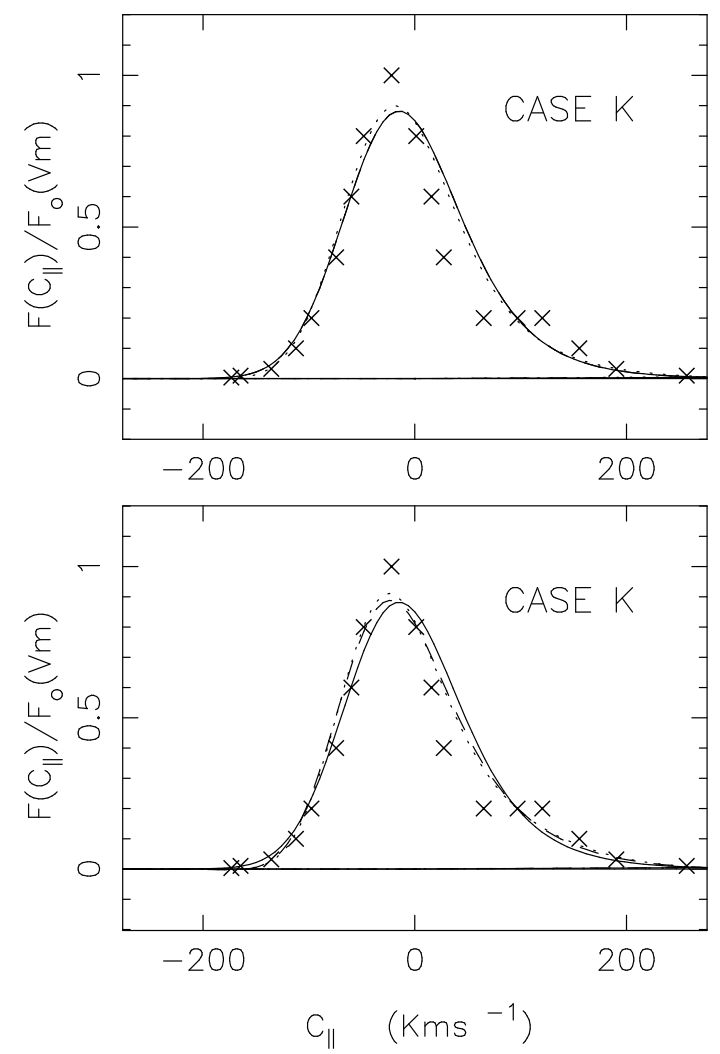

Fig. 6.-Polynomial expansion based on the generalized weight function defined in $\S 4$, centered on the average velocity for case $\mathrm{K}$ issued from Marsch data. The crosses are the Helios probe measurements in the magnetic field direction. The solid line is the zeroth-order function defined in $\S 4$ (see eq. [8]). In the top panel, the dashed line corresponds to order 3 of development (see eq. [11]) and the dotted line to order 4, while the bottom panel shows orders 5 and 7 , respectively.

7 represents the 0,3 , and 4 orders of the generalized polynomial expansion, and the bottom panel represents the orders 0,5 , and 7 .

Comparing Figure 6 to Figure 4, we note that case K seems to be better approached with a bi-Maxwellian polynomial expansion than with a generalized approximation for order $m$ larger than 4. Indeed, in Figure 6, a double peak never appears, but neither do negative values, as observed in Figure 4. Both developments seem to converge slowly and to need orders larger than 3 to fit accurately the observed distribution function.
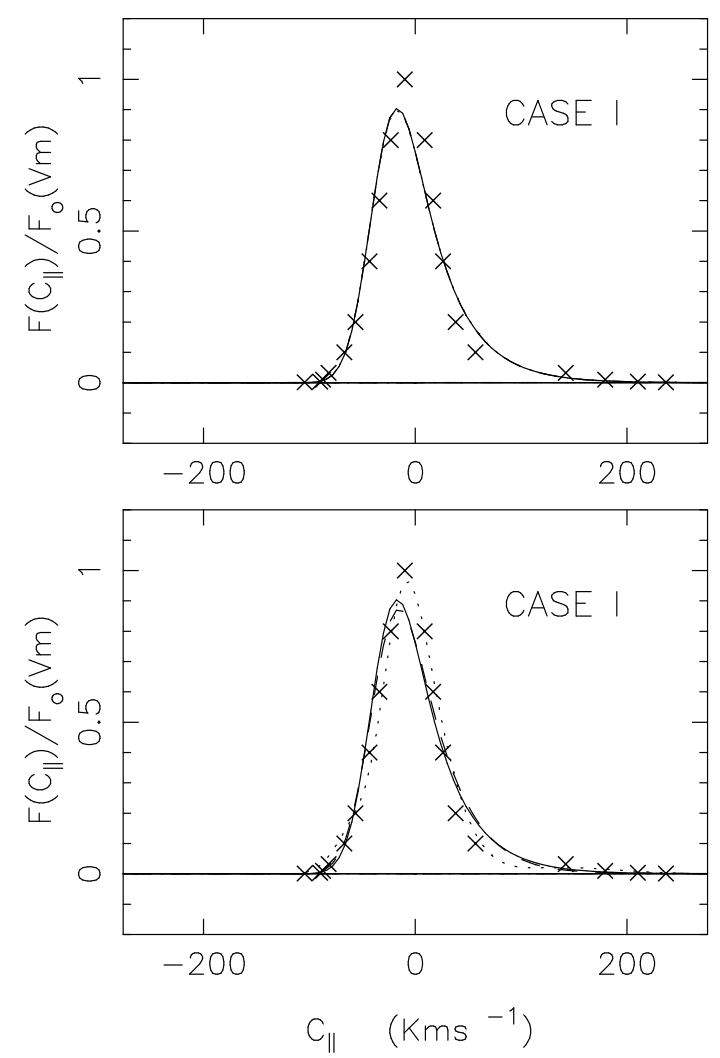

Fig. 7.-Polynomial expansion based on the generalized weight function defined in $\S 4$ for case I: the plotting format is the same as that for Fig. 6.

In case I, the divergence of the bi-Maxwellian polynomial expansion is obvious. With a generalized polynomial expansion the convergence is slow, but with development of order 7 the polynomial expansion approaches very accurately the exact profile.

Another query into the nature of a polynomial expansion $f^{A}$ of given order $p$ is to compare the velocity moments of larger order than $p$ calculated from $f^{A}$, to their exact values.

We have calculated the velocity moments of order 4,5 , and 6 in the magnetic field direction from the biMaxwellian expansion (see eq. [7]) and from the generalized expansion (see eq. [11]) limited at the third order. Table 3 shows the accuracy for cases $\mathrm{I}$ and $\mathrm{K}$ in comparison with the exact values of the velocity moments obtained by

TABLE 3

COMPARISON OF VeLOCITY MOMENTS

\begin{tabular}{|c|c|c|c|c|}
\hline \multirow[b]{2}{*}{ Parameter } & \multicolumn{2}{|c|}{ CASE I } & \multicolumn{2}{|c|}{ Case $\mathrm{K}$} \\
\hline & Generalized & Bi-Maxwellian & Generalized & Bi-Maxwellian \\
\hline$\left(\left\langle c_{\|}^{4}\right\rangle^{A}-\left\langle c_{\|}^{4}\right\rangle^{E}\right) /\left\langle c_{\|}^{4}\right\rangle^{E}(\%) \ldots \ldots$ & 4.7 & 56.2 & 30.5 & 10.5 \\
\hline$\left(\left\langle c_{\|}^{5}\right\rangle^{A}-\left\langle c_{\|}^{5}\right\rangle^{E}\right) /\left\langle c_{\|}^{5}\right\rangle^{E}(\%) \ldots \ldots$ & 19.9 & 35.5 & 89.7 & 39.6 \\
\hline$\left(\left\langle c_{\|}^{6}\right\rangle^{A}-\left\langle c_{\|}^{6}\right\rangle^{E}\right) /\left\langle c_{\|}^{6}\right\rangle^{E}(\%) \ldots \ldots$ & 32.7 & 85.1 & 163 & 17.6 \\
\hline$\left\langle c_{\|}^{4}\right\rangle^{A} /\left[3\left(\left\langle c_{\|}^{2}\right\rangle^{E}\right)^{2}\right] \ldots \ldots \ldots \ldots \ldots$ & 2.21 & $\ldots$ & 1.55 & $\ldots$ \\
\hline$\left\langle c_{\|}^{6}\right\rangle^{A} /\left[15\left(\left\langle c_{\|}^{2}\right\rangle^{E}\right)^{3}\right] \ldots \ldots \ldots \ldots \ldots$ & 9.6 & $\ldots$ & 4.2 & $\ldots$ \\
\hline
\end{tabular}

NoTE.-Comparison between the velocity moments of orders 4,5 , and 6 of the approximation $f^{A}$ (superscript $A$ ) for a polynomial expansion of order 3 (see eqs.[7] and [11]) with respect to the moment value of the exact function (superscript $E$ ) defined in Tables 1 and 2 . The ratios between moments of order 4 and 6 of $f^{A}$ and the corresponding value for a Maxwellian function defined with the exact density and parallel temperature are also given. 
the fitting defined in $\S 2.2$. Their non-Maxwellian nature (in comparison with the theoretical value of the velocity moments of a Maxwellian function defined with the exact parallel temperature) is also provided (last two rows in Table 3). The accuracy of the moment of order 4 is very good in case I for a generalized polynomial expansion and is better estimated than for a bi-Maxwellian one. Furthermore, the study of their non-Maxwellian features allows us to conclude that the velocity moments of higher order than $p$ are more and more "non-Maxwellian" and contribute to the formation of the long tail in the VDF profile. In case K, velocity moments are better estimated with a bi-Maxwellian polynomial expansion despite the negative values of the distribution function. Globally, we can hope for a better macroscopic description of protons in solar wind, because our assumption of velocity moments closure is better adapted to the long-tailed VDF, which is a feature of a large majority of solar wind proton VDF shapes.

\section{DISCUSSION}

We have chosen for our study two typical profiles, cases I and $\mathrm{K}$, from Helios probe measurements. These VDFs reproduce the main features of the proton distribution function observed in the solar wind but are also typical distribution functions of moderate ionized plasmas with a constant collision frequency (Hubert 1985) or with soft interactions (Skullerud 1984) and of Monte Carlo simulations applied to polar wind (Barakat et al. 1995; Wilson 1992). A sum of two Maxwellian functions fits with good precision the cuts through the three-dimensional distribution along the magnetic field direction; in particular, this fitting seems to be well adapted to the long-tailed profiles.

The long-tailed profiles follow in a number of kinetic states an $\exp \left(-\beta v^{\gamma+1}\right)$ law with $\beta$ constant, for instance, in an ion swarm experiment $-0.5 \leq \gamma \leq 1$ (Skullerud 1984) or with $\gamma=0$ for the solution of the BGK equation with a constant collisional frequency (Whealton \& Woo 1971). Then, when these tails are fitted with a sum of two Maxwellian functions [roughly following an $\exp \left(-\beta v^{2}\right)$ law for large velocity values], we overestimate the decreasing of the function. Consequently, the criterion of convergence will be easier to verify. In fact Skullerud (1984) has shown that in the case of a polynomial expansion based on a Maxwellian function, the convergence would never occur if the accurate solution falls off asymptotically slower than any Gaussian. In the same way, Hubert (1985) has clearly shown the difficulty of the convergence of a bi-Maxwellian polynomial expansion. He has constructed such a polynomial expansion to approximate the solution of a BGK equation for the moderately ionized plasmas subjected to an electrostatic field, and he has concluded that whatever the value of $q_{\| p} /\left(v_{\text {th } \|} P_{\| !}\right)_{p}$ might be, the criterion of convergence could not be verified. He has emphasized that higher orders in polynomial expansion do not necessarily give a better approximation.

The slowness of the convergence is another aspect revealed by Figures 4, 5, 6, and 7. In Figures 4 and 5, displaying the bi-Maxwellian polynomial expansion, oscillations and negative values appear as soon as the third order. But they do not appear in Figures 6 and 7 because the zeroth-order generalized function damps such unphysical behaviors. Indeed, in Figure 8 we show the polynomial expansion of order 3 based on the generalized function (see eq. [11]) with a solid line, and on the
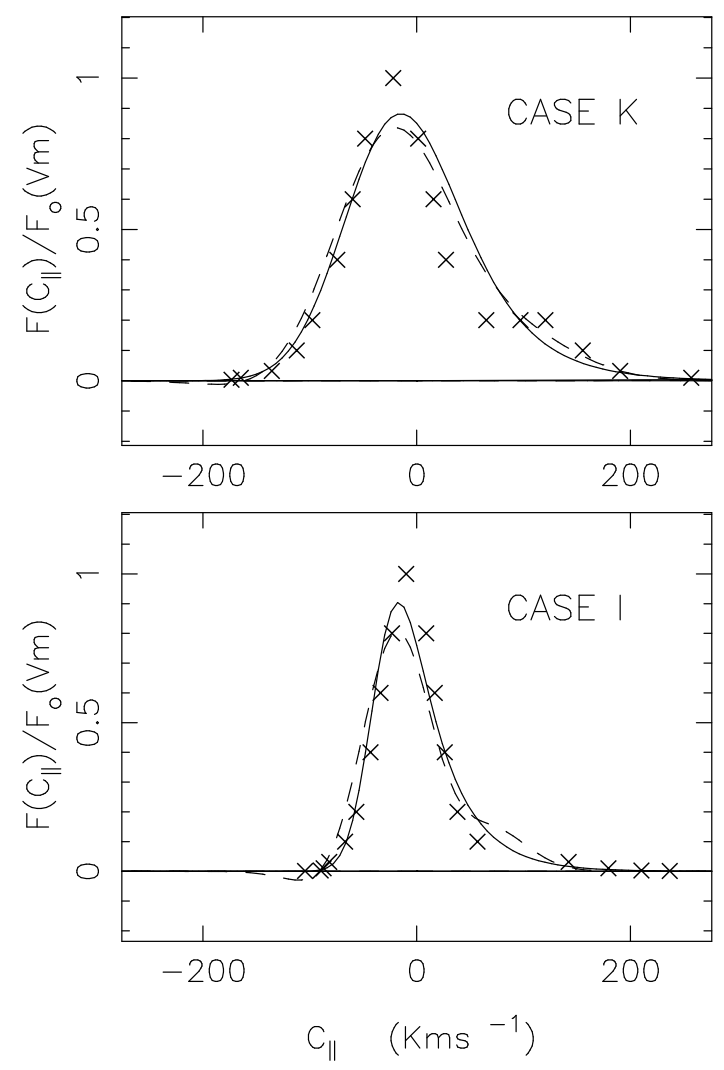

Fig. 8.-Polynomial expansions of order 3 based on the generalized weight function defined in $\S 4$, eq. (11) (solid line), and on the bi-Maxwellian weight function defined in $\S 3$, eq. (7) (dashed line), centered on the average velocity for cases K (top) and I (bottom) issued from Marsch data. The crosses are the Helios probe measurements in the magnetic field direction.

bi-Maxwellian function (see eq. [7]) with a dashed line. We note that the two profiles are very similar. A third-order level of microscopic description cannot reproduce accurately the profiles of the observed VDF. But it is important to stress that the generalized distribution function at the third order does not display negative values, contrary to the bi-Maxwellian polynomial expansion at the same order. This property is certainly important for the evaluation of the velocity collision transfer.

Another point, already emphasized, is the closure of the associated transport equations to a given order. Table 4 shows the precision obtained on the velocity moments of order $p+1$ calculated with an approximated polynomial expansion function of order $p$, for $p$ equal to $3,4,5$, and 6 . There is no evident increase of the accuracy of the derived velocity moments of order $p+1$, except for the biMaxwellian expansion in case K. Nevertheless, we note that there is a good estimation of $\left\langle c_{\|}^{4}\right\rangle$ from the generalized expansion of order 3 in case $I$, as well as for $\left\langle c_{\|}^{5}\right\rangle$ at a fourth order of development. In case $\mathrm{K}$ the results relating to the closure of the transport equations show that a biMaxwellian polynomial expansion seems to be more adapted than a generalized one.

The double-humped functions represent $20 \%$ of all the observations made in the solar wind by the Helios 2 probe according to Marsch et al. (1982). Their real physical origins have not yet been well determined and still constitute an open field of research. Nevertheless, if we want to reproduce their form with a polynomial expansion, then we need to 
TABLE 4

Percentage Error on Velocity Moments

\begin{tabular}{lccccc}
\hline \hline & \multicolumn{2}{c}{ CASE I } & & \multicolumn{2}{c}{ CASE K } \\
\cline { 2 - 3 } \cline { 5 - 6 } Order of Development & Generalized & Bi-Maxwellian & & Generalized & Bi-Maxwellian \\
\hline $3 \ldots \ldots \ldots \ldots \ldots \ldots \ldots \ldots \ldots$ & 4.7 & 56.2 & & 30.5 & 10.5 \\
$4 \ldots \ldots \ldots \ldots \ldots \ldots \ldots \ldots \ldots$ & 21.7 & 35.5 & & 74.1 & 39.6 \\
$5 \ldots \ldots \ldots \ldots \ldots \ldots \ldots \ldots \ldots$ & 42.5 & 27.5 & & 53 & 12 \\
$6 \ldots \ldots \ldots \ldots \ldots \ldots \ldots \ldots$ & 24.7 & 26.5 & & 73 & 7.4 \\
\hline
\end{tabular}

NoTE.-Calculation of the percentage error on velocity moments at order $p+1$ for a biMaxwellian polynomial expansion and a generalized polynomial expansion of order $p=3,4,5$, and 6.

find another weight function and probably at least a fourthorder polynomial expansion. The $f_{G}^{0}$ profile is too different from a double-peaked shape to be well adapted according to Mintzer's advice.

Other approaches exist. For instance, Eu (1980) and Chen \& Eu (1982) have developed a modified moment method in order to reconcile irreversible thermodynamics for system far-from-equilibrium states with the solution of the Boltzmann equation. In this work the microscopic description is defined as $f_{p}(\boldsymbol{r}, \boldsymbol{v}, t)=\exp \left\{-\beta(\boldsymbol{r}, \boldsymbol{v}, t) H_{p}(\boldsymbol{r}, \boldsymbol{v}, t)\right\}$, where $H_{p}$ is a polynomial of third order, and $\beta=1 /\left(k_{B} T_{p}\right)$. To overcome mathematical difficulties in the definition of the entropy density production, the method of cumulant expansion is used (Clause \& Balescu 1982). In a similar attempt Levermore (1995) has suggested the use of distribution functions $f_{p}=\exp \{P(v)\}$, where $P(v)$ is a polynomial of even degree. But Gombosi et al. (1994) has stressed the difficulties in obtaining an explicit closed-form expression of $P$ in terms of the velocity moments of $f_{p}$, for degree of $P$ higher than 2. However, the great interest of Levermore's approach, as in Eu's proposition, is to define an entropy balance equation and to secure the positivity of $f_{p}$ and the realizability criterion. We have tried to verify whether such a function defined with a polynomial $P$ of degree 4 (the lowest order to model the double-humped function) was able to fit cases I and $\mathrm{K}$. This was easily obtained in case $\mathrm{K}$, but without good precision, and we have met difficulties in fitting profile case I. Another method has been proposed by Cuperman, Weiss, \& Dryer (1983) and Cuperman et al. (1987). They have constructed a VDF from the minimization of Boltzmann's $H$-function, subjected to the constraints provided by the set of the selected components of the approximation (seven or nine macroscopic parameters). Although applied to spherically symmetric systems, this model provides almost all the typical profiles of the solar wind and is able, according to the authors, to generate an improved associated system of transport equations. The sum of two Maxwellian functions in the velocity space parallel to the magnetic field, multiplied by a Maxwellian function in the perpendicular velocity space, should also be considered. But it remains difficult to use it because the derived system of transport equations is composed of 57 variables, as the velocity moments up to order 5 are needed to define the sum of two Maxwellian functions.

\section{CONCLUSION}

The observations of the Helios probe in solar wind between 0.3 and $1 \mathrm{AU}$ have revealed the main aspects of the proton VDF: a magnetic field-aligned suprathermal tail and, for about $20 \%$ of them, the presence of a second peak. These nonthermal features have been studied by several authors. Up to now, the most sophisticated solution proposed to model these proton VDFs is a polynomial expansion with a bi-Maxwellian as the weight factor, associated with a system of transport equations constructed following the Grad method. This model provides temperature anisotropies similar to observations, contrary to the collisionless approaches or to the fluid models.

But the choice of the microscopic description is not satisfied according to criticisms that have determined the field of application of such a model. In fact, the polynomial expansion is able to model only exact solutions that are near the weight function. We have established that the 16 moment bi-Maxwellian polynomial expansion does not converge for the most typical case of a proton VDF in solar wind. In this case, the system of transport equations does not respect mathematical rules such as the hyperbolicity condition. Moreover, the limited order polynomial expansion can generate negative values. Therefore, the collisional terms are not well estimated, and the closure assumption is not appropriate. We have also shown that an increase of the order of development does not improve the model.

Therefore, we propose a new approach to model the proton microscopic state in the solar and polar winds (or, more generally, for stellar atmosphere expansion) and to derive generalized transport equations. It is also a polynomial expansion, but it is based on a function derived from the exact solution of the BGK equation for the moderate ionized plasma subjected to an electrostatic field. The advantage of this representation is an intrinsic asymmetry of the weight function, which displays a suprathermal tail in the magnetic field direction, that is, a profile close to $80 \%$ of the observed VDF. Furthermore, the heat flux $q_{\| p}$ that is used in the definition of the weight function is not involved in the construction of the polynomial part of the expansion. Therefore, the limitation on the intensity of $q_{\| p}$ derives only from the rules of construction of this generalized weight function. But in the solar wind it seems not to be a difficulty because almost all the observations are in agreement with this limitation. Consequently, no negative value of the VDF appears with large values of the parallel heat flux, as it was the case with a bi-Maxwellian polynomial expansion of order 3. This new solution verifies the Mintzer criterion of convergence in all the observed typical cases, contrary to the bi-Maxwellian polynomial expansion, and, consequent$1 y$, it is better adapted for the determination of macroscopic parameters when we solve the associated system of transport equations. 
The double-humped VDF needs to use more sophisticated zeroth-order models for a multimoment approach or kinetic models such as those in Livi \& Marsch (1987). Indeed, these authors have combined the action of the large-scale interplanetary magnetic field and of the col- lisional scattering and have obtained double-humped profiles.

Next developments will be the construction of the set of transport equations associated with the generalized polynomial expansion and the study of its properties.

\section{REFERENCES}

Abramovitz, M., \& Stegun, I. A. 1964, Handbook of Mathematical Functions (New York: Dover)

Barakat, A. R., Bargouthy, I. A., \& Schunk, R. W. 1995, Geophys. Res. Lett., 22, 1857

Barakat, A. R., \& Hubert, D. 1990, Ann. Geophys., 8, 697

Chamberlain, J. W. 1960, ApJ, 131, 47

Chapman, S. 1965, Smithson. Contrib. Astrophys., 2, 1

Chen, M., \& Eu, B. C. 1982, J. Chem. Phys., 77, 2696

Chew, G. F., Goldgerger, M. L., \& Low, F. E. 1956, Proc. R. Soc. London A, 236, 112

Chodura, R., \& Pohl, F. 1971, Plasma Phys., 13, 645

Clause, P.-J., \& Balescu, R. 1982, Plasma Phys., 24, 1429

Cordier, S. 1994a, Math. Models Meth. Appl. Sci., 4, 625 1994b, Math. Models Meth. Appl. Sci., 4, 647

Cuperman, S., Weiss, I., \& Dryer, M. 1980, ApJ, 239, 345 . 1981, ApJ, 251, 297 1983, ApJ, 273, 363

Cuperman, S., Yatom, H., Dryer, M., \& Lewis, D. 1987, ApJ, 314, 404

Demars, H. G., \& Schunk, R. W. 1979, J. Phys. D, 12, 1051 - 1989, Planet. Space Sci., 37, 85 . 1990, Planet. Space Sci., 38, 1091

. 1991, Planet. Space Sci., 39, 435

Eu, B. C. 1980, J. Chem. Phys., 73, 2958

Feldman, W. C. 1979, Sol. Syst. Plasma Phys., 1, 321

Gazis, P. R. 1984, J. Geophys. Res., 89,775

Gombosi, T. I., Groth, C. P. T., Roe, P. L., \& Brown, S. L. 1994, Phys. Fluids, submitted

Gombosi, T. I., \& Rasmussen, C. E. 1991, J. Geophys. Res., 96, 7759

Grad, H. 1958, Handb. Phys., 12

Hubert, D. 1983, Planet. Space Sci., 31, 119

-.1985, J. Phys. D, 18, 1521
Hubert, D., \& Barakat, A. R. 1990, Ann. Geophys., 8, 687

Jancel, R., \& Kahan, J. 1966, Electrodynamics of Plasma (New York: Wiley)

Lemaire, J., \& Scherer, M. 1971, J. Geophys. Res., 76, 7479

. 1973, Rev. Geophys. Space Phys., 11, 427

Levermore, C., Morokoff, W. J., \& Nadiga, B. T. 1995, Phys. Fluids, submitted

Levermore, C. D. 1995, J. Stat. Phys., submitted

Liu, S., Marsch, E., Livi, S., Woch, J., Wilken, B., von Steiger, R., \& Goeckler, G. 1995, Geophys. Res. Lett., 22, 2445

Livi, S., \& Marsch, E. 1987, J. Geophys. Res., 92, 7255

Lowell, H. H., Jr. 1967, Phys. Fluids, 10, 35

Maksimovic, M. 1995, Ph.D. thesis, Univ. Paris VII

Marsch, E. 1982, NASA CP Sol. Wind, 5, 355

Marsch, E., Mulhauser, K. H., Schwenn, R., Rosenbauer, H., Pillip, W., \& Neubauer, F. M. 1982, J. Geophys. Res., 87, 52

Mintzer, D. 1965, Phys. Fluids, 8, 1076

Montgomery, M. D., Gary, S. P., Feldman, W. C., \& Forslund, D. W. 1976, J. Geophys. Res., 81, 2743

Oraevskii, V., Chodura, R., \& Feneberg, W. 1968, Plasma Phys., 10, 819

Palmadesso, P. J., Ganguli, S. B., \& Mitchell, H. G., Jr. 1988, Geophys. Monogr. Ser., 44, 133

Parker, E. N. 1958, ApJ, 128, 664

Robineau, A., Blelly, P.-L., \& Fontanari, J. 1996, J. Atmos. Terr. Phys. 58, 257

Schwenn, R., \& Marsch, E., eds. 1991, Physics of Inner Heliosphere, Part 2: Particles, Waves, and Turbulence (Berlin: Springer)

Shizgal, B. 1977, Planet. Space Sci., 25, 203

Skullerud, H. R. 1984, J. Phys. B, 17, 913

Whealton, J. H., \& Woo, S.-B. 1971, Phys. Rev. A, 6, 2319

Wilson, G. R. 1992, J. Geophys. Res., 97, 10,551 